\title{
Diversidade de mesoinvertebrados associada ao tratamento aeróbio de resíduos
}

\section{sólidos orgânicos domiciliares}

\author{
Diversity of mesoinvertebrates associated with aerobic treatment of home organic solid waste \\ Diversidad de mesoinvertebrados asociados al tratamiento aeróbico de residuos sólidos orgánicos \\ domiciliarios
}

Recebido: 19/01/2021 | Revisado: 21/01/2021 | Aceito: 25/01/2021 | Publicado: 31/01/2021

Elaine Cristina dos Santos Araújo
ORCID: https://orcid.org/0000-0002-6288-8469
Universidade Federal de Campina Grande, Brasil
E-mail: cryslainne@gmail.com
Monica Maria Pereira da Silva
ORCID: https://orcid.org/0000-0002-1593-1698
Universidade Federal de Campina Grande, Brasil
E-mail: monicaea@ terra.com.br
Adriana Veríssimo da Silva
ORCID: https://orcid.org/0000-0001-7695-284X
Universidade Estadual da Paraíba, Brasil
E-mail: verissimo-pb@ hotmail.com
Adrianne Teixeira Barros
ORCID: https://orcid.org/0000-0002-7393-642X
Universidade Federal de Campina Grande, Brasil
E-mail: adriannebarros@yahoo.com.br
Valderi Duarte Leite
ORCID: https://orcid.org/0000-0001-5861-7407
Universidade de São Paulo, Brasil
E-mail: mangabeiraleite@ gmail.com

\section{Resumo}

Os mesoinvertebrados provocam mudanças nas propriedades físicas e químicas da matéria orgânica durante o processo de compostagem. São excelentes recicladores de matéria orgânica. O objetivo deste estudo foi avaliar a densidade e a diversidade de mesoinvertebrados em sistema de tratamento aeróbio de resíduos sólidos orgânicos domiciliares. O trabalho realizado em Campina Grande, estado da Paraíba, Brasil, seguiu os princípios da pesquisa experimental. Foram coletados resíduos sólidos orgânicos em 46 residências, previamente segregados na fonte geradora. Por 15 semanas o sistema foi monitorado diariamente. Durante a compostagem foram coletados 1.258 indivíduos pertencentes à seis ordens: Diptera (88,5\%), Mesostigmata (5,5\%), Coleoptera $(2,4 \%)$, Isopoda $(2,4 \%)$, Araneae $(0,6 \%)$ e Orthoptera $(0,6 \%)$. No processo de compostagem foram observadas três fases distintas, nas quais as espécies foram diferenciadas. Constatou-se que no sistema de tratamento dos resíduos sólidos orgânicos domiciliares, há considerável participação de organismos que atuam nas diferentes fases do tratamento, correlacionando-se com as mudanças físico-químicas e influenciando no funcionamento de todo o sistema.

Palavras-chave: Meio ambiente; Tratamento biológico; Gestão ambiental; Biodiversidade.

\begin{abstract}
The mesoinvertebrates cause changes in the physical and chemical properties of organic matter during the composting process. They are excellent recyclers of organic matter. The aim of this study was to evaluate the density and diversity of mesoinvertebrates in an aerobic treatment system for home organic solid waste. The work carried out in Campina Grande, state of Paraíba, Brazil, followed the principles of experimental research. Solid organic waste was collected in 46 residences, previously segregated in the generating source. For 15 weeks the system was monitored daily. During composting, 1.258 individuals belonging to six orders were collected: Diptera (88.5\%), Mesostigmata (5.5\%), Coleoptera $(2.4 \%)$, Isopoda (2.4\%), Araneae (0.6\%) and Orthoptera (0.6\%). In the composting process, three distinct phases were observed, in which the species were differentiated. It was found that during the treatment system for household solid organic waste, there is considerable participation of organisms that act in the different phases of treatment, correlating with physical-chemical changes and influencing the functioning of the entire system.
\end{abstract}

Keywords: Environment; Biological treatment; Environmental management; Biodiversity. 


\section{Resumen}

Los mesoinvertebrados provocan cambios en las propiedades físicas y químicas de la materia orgánica durante el proceso de compostaje. Son excelentes recicladores de materia orgánica. El objetivo de este estudio fue evaluar la densidad y diversidad de mesoinvertebrados en un sistema de tratamiento aeróbico de residuos sólidos orgánicos domiciliarios. El trabajo realizado en Campina Grande, estado de Paraíba, Brasil, siguió los principios de la investigación experimental. Los residuos orgánicos sólidos se recolectaron en 46 residencias, previamente segregadas en la fuente generadora. Durante 15 semanas, el sistema se controló diariamente. Durante el compostaje se recolectaron 1.258 individuos pertenecientes a seis órdenes: Diptera (88.5\%), Mesostigmata (5.5\%), Coleoptera $(2.4 \%)$, Isopoda $(2.4 \%)$, Araneae $(0.6 \%)$ ) y Orthoptera (0.6\%). En el proceso de compostaje se observaron tres fases distintas, en las que se diferenciaron las especies. Se encontró que durante el sistema de tratamiento de residuos orgánicos sólidos domiciliarios, existe una participación considerable de organismos que actúan en las diferentes fases del tratamiento, correlacionándose con los cambios físico-químicos e influyendo en el funcionamiento de todo el sistema.

Palablas clave: Medio ambiente; Tratamiento biológico; Gestión ambiental; Biodiversidad.

\section{Introdução}

Um dos grandes problemas ambientais enfrentados atualmente pela humanidade é a geração de resíduos, tendo em vista os impactos negativos acarretados aos sistemas ambientais, econômicos e sociais. O cenário demanda tomada de decisão dos gestores, no sentido de adotar um conjunto de alternativas voltado para prevenir, mitigar ou mesmo, evitar esses impactos (Silva et al., 2020).

Dentre os resíduos sólidos urbanos, cerca de 46\% correspondem aos orgânicos (Kaza et al., 2018), contudo, menos de 13\% dos resíduos sólidos de origem orgânica são coletados separadamente e tratados em todo mundo (Jürgensen; Gilbert \& Ramola, 2020). Um potencial significativo de resíduos sólidos orgânicos gerados é disposto de forma inadequada, provocando à formação de odores putrescíveis, liberação de gases do efeito estufa na atmosfera e a contaminação e poluição ambiental (Gilbert; Jürgensen \& Ramola, 2020).

A parcela orgânica representa um risco de Saúde Pública, sobretudo, ao ponderar que a composição da parcela orgânica detém condições favoráveis para abrigar diferentes organismos, dentre esses, organismos com significativa carga patogênica, a exemplo de enterobactérias, fungos, protozoários e helmintos e vírus, como o novo coronavírus; todos causadores de doenças resultantes da insalubridade ambiental (Freitas et al., 2020; Silva et al., 2020; Silva et al. 2020a).

O substrato em processo de compostagem constitui um ambiente com condições favoráveis para alimentação, deposição de ovos e desenvolvimento de larvas de uma comunidade dinâmica de organismos, dentre os quais, os mesoinvertebrados (Mira \& Jaramillo, 2010). Esses organismos decompõem a matéria orgânica em partículas menores, aumentando assim, a área de superfície para a ação microbiana, tornando-os excelentes recicladores de matéria orgânica, uma vez que, ao digerirem o substrato que constituem o sistema, transformam e liberam nutrientes (Steel \& Bert, 2011), possibilitando, desse modo, a ciclagem da matéria.

Uma das alternativas mais viável ao tratamento e destinação final adequadas dos resíduos sólidos orgânicos é a compostagem, conceituada na Resolução n 481 de 03 de outubro de 2017, do CONAMA (Conselho Nacional do Meio ambiente), como um processo de decomposição biológica, em condições aeróbias e controladas, efetuado por uma população diversificada de organismos, resultando em composto estabilizado (Brasil, 2017) e higienizado.

A compostagem é representada pela atividade combinada de uma ampla sucessão de organismos coexistindo no mesmo ambiente, um se sobrepondo ao outro e cada um emergindo gradualmente à medida que as condições se tornam favoráveis, resultando em mudanças contínuas de parâmetros físicos e químicos no substrato (Polprasert \& Koottatep, 2017).

Através deste processo de degradação biológica ocorrem à eliminação de organismos patógenos, sementes e ervas daninhas, a recuperação de nutrientes que podem ser utilizados no solo e reduzir erosão, a acidez, além de melhorar o escoamento superficial e de beneficiar a produtividade em hortas e jardins (Araújo et al., 2019). 
Há necessidade de criar condições favoráveis à ação dos organismos aeróbios, uma vez que a natureza do organismo ou da comunidade, o número de espécies e a intensidade da atividade de decomposição dependem dessas condições (Polprasert \& Koottatep, 2017). Quando o sistema de compostagem é mal projetado ou não há controle eficiente dos parâmetros químicos e físicos, o sistema pode ser um fator limitante à sucessão ecológica dos organismos, influenciando diretamente na qualidade final do composto (Araújo et al., 2019).

Dessa forma, para obter um composto orgânico sanitizado e higienizado, os organismos devem encontrar na compostagem, um nicho ecológico adequado, ao qual disponha de recursos necessários para sua existência e de seus descendentes, como espaço, habitat, alimento, água e local para descarte dos resíduos (Madigan et al., 2016).

Entende-se que a ação dos organismos aeróbios durante o tratamento da parcela orgânica dos resíduos sólidos em sistema de compostagem, expressa as relações de um sistema ecológico, logo, ocorrem, dentre outros fatores, as cadeias tróficas, o ciclo da matéria, o fluxo de energia, sucessão ecológica e as atividades que permitem a homeostase do próprio sistema (Araújo et al., 2019). Dessa forma, a compostagem favorece a continuidade das espécies especializadas na degradação da matéria orgânica, ao mesmo tempo em que soluciona um dos principais problemas que concorrem para o desequilíbrio ambiental (Nascimento et al., 2017; Polprasert \& Koottatep, 2017; Araújo et al., 2019; Gilbert; Ricci-Jürgensen \& Ramola, 2020; Silva et al., 2020a).

A densidade e a diversidade de mesoinvertebrados estão relacionadas à sua dieta, às características físicas e químicas e às condições ambientais. A observação e o acompanhamento dos mesoinvertebrados podem ser um indicativo da estabilização do composto (Morales \& Wolff, 2010).

Nessa perspectiva, objetivo desse trabalho foi avaliar a densidade e a diversidade de mesoinvertebrados em sistemas de tratamento aeróbio de resíduos sólidos orgânicos domiciliares.

\section{Metodologia}

\subsection{Caracterização da pesquisa e procedimento metodológico}

O sistema de tratamento de resíduos sólidos orgânicos foi instalado nas dependências da UEPB (Universidade Estadual da Paraíba), em Campina Grande, estado da Paraíba, Brasil. Na cobertura do sistema foi utilizada estrutura em madeira, lonas plásticas e telas de proteção, com intuito de reduzir os fatores ambientais intensos, decorrentes das chuvas no período noturno e a radiação solar excessiva durante os dias (Figura 1), típicos do período em que foi instalado o sistema de compostagem (agosto e novembro) no Nordeste do Brasil.

Figura 1. Desenho e foto do sistema de compostagem.

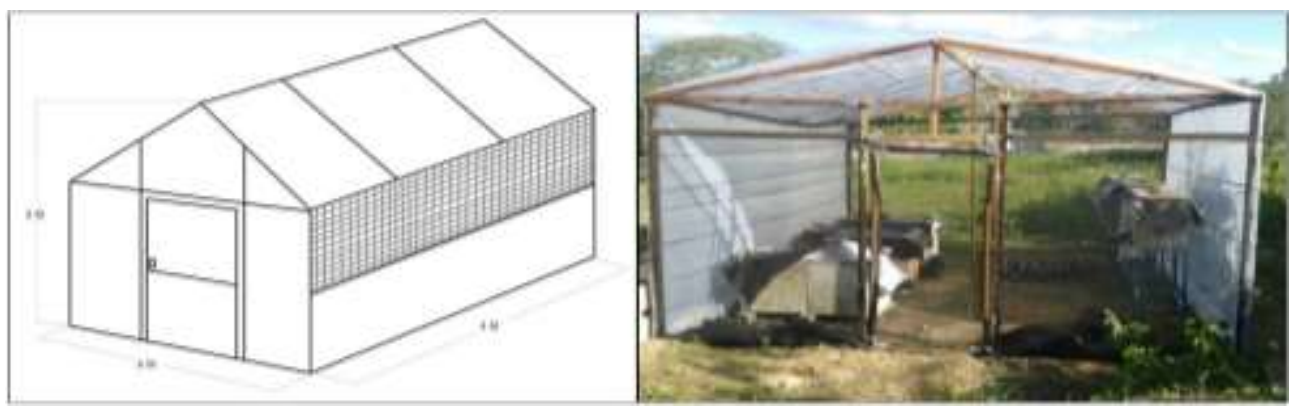

Fonte: Araújo (2018). 
O sistema de tratamento de resíduos sólidos orgânicos foi composto por seis composteiras móveis. Estas podem ser realocadas no final do experimento (Figura 2). As composteiras utilizadas no experimento consistiram de dois tipos de tratamentos (CCR - composteira de concreto retangular e CAR - composteira de aço inoxidável) com três repetições (CCR1; CCR2 e CCR3; CAR1, CAR2 e CAR3).

Figura 2. Composteiras móveis utilizadas no sistema de compostagem. Foto A: Composteira de concreto retangular (CCR). Foto B: Composteiras de aço inoxidável (CAR).
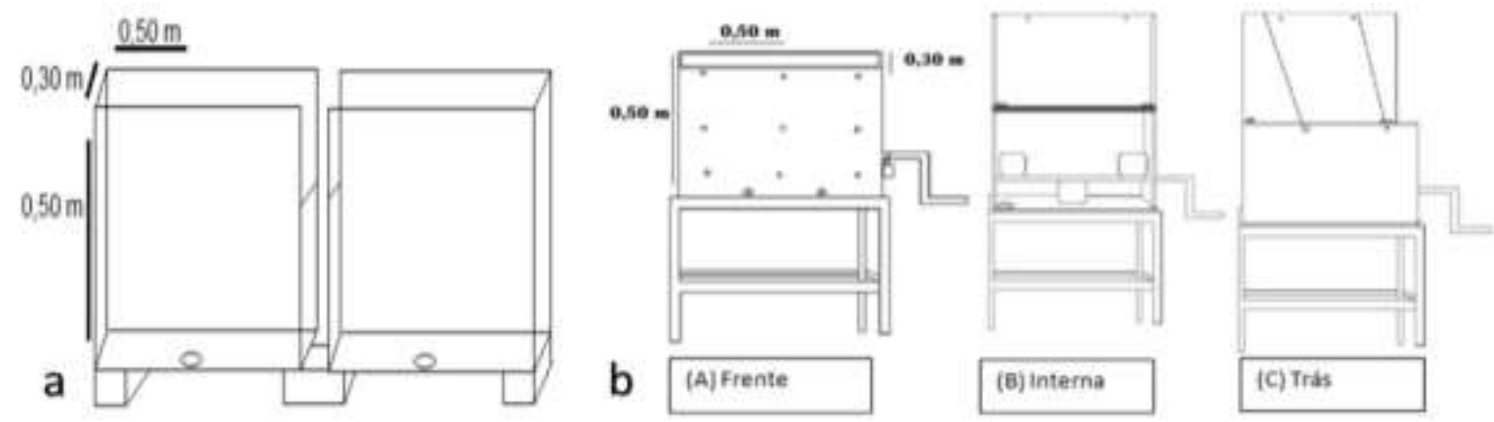

Fonte: Nascimento (2015).

A composteira de concreto retangular (CCR) foi formada por dois compartimentos, com redução de $0,25 \mathrm{~m}$ em uma das laterais de cada compartimento, para facilitar o reviramento manual entre elas. As composteiras de concreto retangular ficaram suspensas em blocos de concreto a $0,5 \mathrm{~cm}$ para evitar o contato com a superfície. $\mathrm{O}$ subsistema de composteiras de aço inoxidável (CAR) foi construído com um único compartimento com manivela móvel acoplada à parede lateral para o reviramento do cossubstrato. A composteira era suspensa em base de ferro 1,0 m de altura (Nascimento, 2015; Araujo, 2018).

Para evitar a atração de insetos noturnos, as composteiras detinham cobertura completa com tela de nylon.

Cada composteira foi alimentada com $20 \mathrm{~kg}$ de substrato, sendo $80 \%$ constituídos de resíduos sólidos orgânicos domiciliares e $20 \%$ de estruturantes. O estruturante correspondeu aos resíduos de folhas, flores e materiais não degradados em outros sistemas de compostagem, rejeito e farelo.

Os resíduos sólidos orgânicos domiciliares e resíduos de folhas foram coletados nas residências de moradores que realizavam a coleta seletiva no bairro de maior população da cidade de Campina Grande-PB, Malvinas. Os resíduos de flores foram coletados de eventos realizados na Matriz da Paróquia Jesus Libertador, localizada no referido bairro. A coleta foi feita pelos líderes comunitários que armazenavam e depois repassavam à equipe técnica (Araújo et al., 2019).

Os resíduos sólidos orgânicos domiciliares, as folhas e os estruturantes foram despejados em lona plástica e misturados para coleta de amostras para as análises laboratoriais, em seguida, todo o substrato foi pesado e usado para alimentar as respectivas composteiras.

O sistema foi avaliado e monitorado por 15 semanas, tempo demandado para estabilização e sanitização dos resíduos sólidos organicos, atingindo-se assim, o seu tratamento. Neste período, duas vezes por semana eram coletadas amostras de $25 \mathrm{~g}$ de substrato para análise de mesoinvertebrados. Essa amostra proposta por Silva (2008) se mostrou adequada à identificação de mesoinvertebrados por possibilitar a observação de maior número de indivíduos. As coletas aconteciam durante a aeração dos sistemas. De cada composteira, era retirada uma amostra composta por dez subamostras obtidas de diferentes estratos (base, centro e superfície).

Para a quantificação e identificação dos mesoinvertebrados presente nas diferentes fases do processo de compostagem foi adotado o método de captura manual, catação, identificação e contagem (CIC), proposto por Silva (2008). A 
amostra era colocada em placa de Petri; com auxílio de uma lupa binocular foi feita identificação e contagem (CIC) dos mesoinvertebrados presentes em cada amostra, de acordo com a metodologia sugerida por Silva (2008). Para identificação taxonômica foram utilizadas as chaves de identificação de McAlpine et al. (1987), Sterh (1991) e Borror et al. (1992).

\subsection{Análise dos dados}

A análise da correlação entre os mesoinvertebrados e os parâmetros físico e químicos estudados nos sistemas CCR e CAR foi avaliada por meio do teste de Pearson através do Software Microsoft Excel (Quadro 1).

Quadro 1. Coeficiente de correlação (r).

\begin{tabular}{|cc|}
\hline Correlação & Valor de $\mathbf{r}$ \\
\hline Bem fraca & 0,00 a 0,19 \\
Fraca & 0,20 a 0,39 \\
Moderada & 0,40 a 0,69 \\
Forte & 0,70 a 0,89 \\
Muito forte & 0,90 a 1,00 \\
\hline
\end{tabular}

Fonte: Shimakura (2006).

Segundo esse teste, quando o coeficiente de correlação é igual a um $(\mathrm{r}=1)$ há uma correlação perfeita positiva entre duas variáveis. Enquanto, o coeficiente de correlação igual a menos um $(r=-1)$ pressupõe a correlação negativa perfeita entre duas variáveis, e o coeficiente igual à zero, indica que as duas variáveis não são dependentes (Shimakura, 2006).

Para avaliação da densidade dos mesoinvertebrados nos sistemas, foram realizadas comparações das comunidades no período estudado em relação à quantidade de sólidos totais da amostra. A densidade de mesoinvertebrados para cada tratamento foi determinada de acordo com a Fórmula 1.

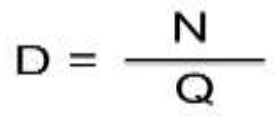

Sendo:

$\mathrm{n}$ = número de indivíduos;

$\mathrm{q}=$ quantidade de sólidos total da amostra.

Os dados contidos neste trabalho são apresentados tomando-se por base a média aritmética entre as repetições de cada tratamento, uma vez que não foi identificada diferença estatística significante entre as repetições (CCR1, CCR2 e CCR3; CAR1, CAR2 e CAR3).

Para a organização dos dados foi utilizado o Software do Microsoft Office Excel 2017. O uso de tabela foi priorizado para melhor interpretação e visualização dos dados. 


\section{Resultados e Discussão}

\subsection{Táxons coletados no sistema}

Durante o experimento de compostagem dos resíduos sólidos orgânicos domiciliares, foram coletados 1.258 indivíduos pertencentes a seis ordens. Na ordem Diptera foram coletados 1.114 indivíduos $(88,5 \%)$, pertencentes aos táxons Ornidia obesa, Fannia canicularis, Drosophila sp., Hermetia illucens e Musca domestica; na ordem Orthoptera foram 8 indivíduos $(0,6 \%)$ pertencentes ao gênero Achaeta sp.; na ordem Coleoptera foram 30 indivíduos $(2,4 \%)$ das famílias Staphylinidae e Hybosoridae; entre os membros da ordem Isopoda foram coletados 30 indivíduos $(2,4 \%)$, representados pelo gênero Armadillidium sp.; na ordem Mesostigmata foram 69 indivíduos (5,5\%) da família Parasitidae; enquanto que na ordem Araneae foram 7 indivíduos $(0,6 \%)$, pertencentes à família Theridiosomatidae (Figura 3).

Figura 3. Mesoinvertebrados encontrados nos sistemas de compostagem. Foto A: forma larval de Musca domestica; Foto B: Armadillidium sp.; Foto C: Ornidia obesa; Foto D: Estágios de Hermetia illucens.

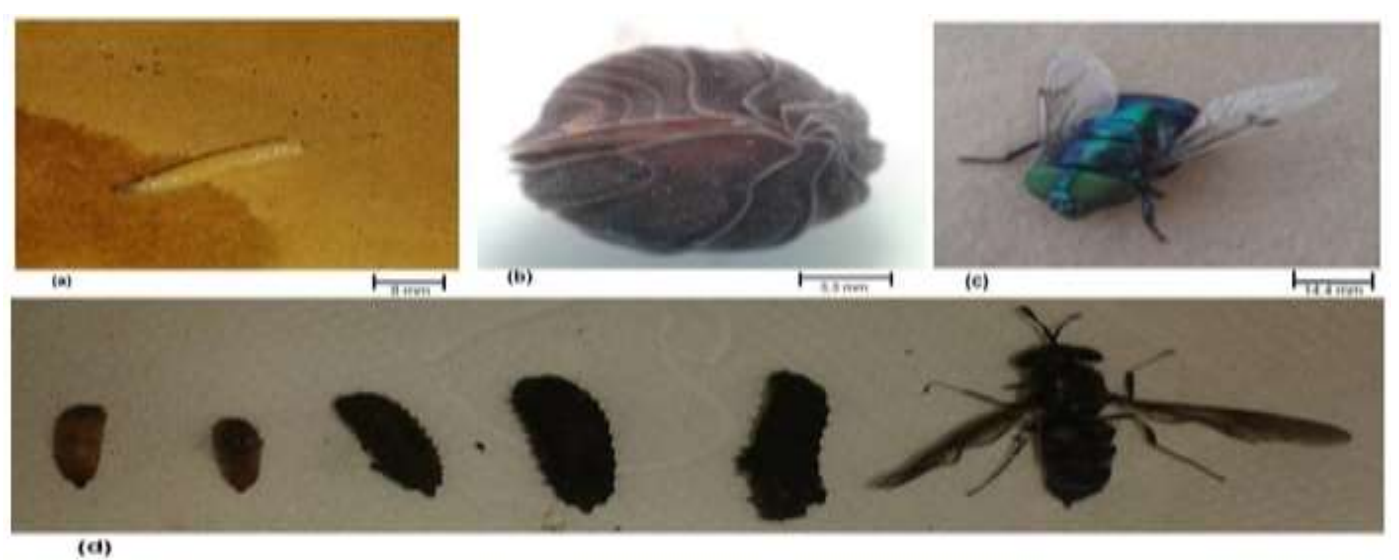

Fonte: Araújo (2018).

\subsection{Densidade e diversidade de mesoinvertebrados}

Verificou-se que a densidade média de mesoinvertebrados identificada nos sistemas estudados variou em relação ao tempo, alcançando maior densidade em períodos diferentes para cada tratamento (Tabela 1). A densidade biológica máxima dos indivíduos presentes nos sistemas de compostagem, variou de 2,41 indivíduos/gST no sistema de composteiras de concreto retangular (CCR) a 1,92 indivíduos/gST no sistema de composteiras de aço inoxidável retangular (CAR).

No sistema CCR, conforme Tabela 1, constataram-se as maiores densidades entre a fase termófila (níveis de temperatura de 45 a $50{ }^{\circ} \mathrm{C}$ ) e o início da fase de resfriamento (níveis de temperatura de $45{ }^{\circ} \mathrm{C}$ a temperatura ambiente), alcançando-se valores elevados até o $54^{\circ}$ dia. Esse período foi característico por apresentar mudanças consideráveis nas condições físicas e químicas do substrato. O dia de maior densidade de mesoinvertebrados foi o $9^{\circ}$, período de alto teor de umidade $(69,4 \%)$, material orgânico complexo, temperaturas elevadas $\left(40,2{ }^{\circ} \mathrm{C}\right)$ e $\mathrm{pH}$ alcalino $(8,3)$. Nessa fase foram observadas, principalmente, espécies da ordem Díptera. Embora tenha apresentado alta quantidade de táxons, não foi necessariamente o dia de maior diversidade de mesoinveterbrados $(\mathrm{N}=7)$, apresentado apenas espécies de dípteros e coleópteros, possivelmente, em virtude da faixa de tolerância de alguns organismos às condições físicas e químicas.

No sistema CCR, de acordo com os dados apresentados na Tabela 1, houve redução gradativa da densidade a partir do $58^{\circ}$ dia até alcançar valores poucos significativos, contudo, no $72^{\circ}$ dia, observou-se um aumento considerável na densidade de mesoinvertebrado em virtude da presença de adultos da espécie Hermetia illucens sobre o substrato, apontando-se que em sua 
maioria, a espécie não sai do sistema na fase adulta, podendo serem coletadas nas retiradas das amostras. A permanência do adulto nos sistemas de compostagem é justificada devido às fêmeas se encontrarem em repouso para atraírem os machos em pleno voo, conforme citam Tingle et al. (1975).

No sistema CAR foram constatados menores valores de densidade e diversidade de mesoinvertebrados em comparação ao tratamento CCR (Tabela 1). Este fato, possivelmente, é resultado da geração de chorume no sistema entre os dias $9^{\circ}$ e $16^{\circ}$, embora em quantidade mínima, o que pode ter procrastinado a incidência dos mesoinvertebrados, ratificando que a estrutura da composteira tem influência direta no desenvolvimento e na ação dos organismos que participam da compostagem.

Tabela 1. Valores médios referentes a mesoinvertebrados nos tratamentos de compostagem de resíduos sólidos orgânicos domiciliares. Campina Grande, estado da Paraíba, Brasil. Agosto a novembro de 2017.

\begin{tabular}{|c|c|c|c|c|}
\hline \multirow{3}{*}{$\begin{array}{l}\text { Tempo } \\
\text { (dia) }\end{array}$} & \multicolumn{4}{|c|}{ Mesoinvertebrados } \\
\hline & \multicolumn{2}{|c|}{ CCR } & \multicolumn{2}{|c|}{ CAR } \\
\hline & $\begin{array}{c}\text { Densidade } \\
\text { (ind/gST) }\end{array}$ & $\begin{array}{l}\text { Diversidade } \\
\text { (nº de táxons) }\end{array}$ & $\begin{array}{r}\text { Densidade } \\
\text { (ind/gST) }\end{array}$ & $\begin{array}{l}\text { Diversidade } \\
\text { (no de taxons) }\end{array}$ \\
\hline $1^{\circ}$ & 0 & 0 & 0 & 0 \\
\hline $5^{\circ}$ & 1,08 & 3 & 0,13 & 2 \\
\hline $9^{\circ}$ & 2,41 & 7 & 1,16 & 3 \\
\hline $12^{\circ}$ & 1,97 & 4 & 1,33 & 6 \\
\hline $16^{\circ}$ & 1,93 & 7 & 1,92 & 5 \\
\hline $19^{\circ}$ & 1,54 & 8 & 1,07 & 6 \\
\hline $23^{\circ}$ & 1,35 & 3 & 0,66 & 4 \\
\hline $26^{\circ}$ & 1,61 & 4 & 1,22 & 6 \\
\hline $30^{\circ}$ & 1,42 & 8 & 0,98 & 7 \\
\hline $33^{\circ}$ & 1,09 & 5 & 0,53 & 5 \\
\hline $37^{\circ}$ & 1,05 & 6 & 0,29 & 5 \\
\hline $40^{\circ}$ & 1,73 & 4 & 0,47 & 6 \\
\hline $44^{\circ}$ & 1,34 & 6 & 0,32 & 4 \\
\hline $47^{\circ}$ & 1,03 & 3 & 0,32 & 5 \\
\hline $51^{\circ}$ & 1,48 & 4 & 0,44 & 5 \\
\hline $54^{\circ}$ & 1,44 & 2 & 0,2 & 3 \\
\hline $58^{\circ}$ & 0,87 & 3 & 0,41 & 2 \\
\hline $61^{\circ}$ & 0,56 & 3 & 0,37 & 2 \\
\hline $65^{\circ}$ & 0,42 & 2 & 0,47 & 2 \\
\hline $68^{\circ}$ & 0,2 & 2 & 0,27 & 2 \\
\hline $72^{\circ}$ & 1,09 & 6 & 0,1 & 2 \\
\hline $75^{\circ}$ & 0,67 & 2 & 0,11 & 3 \\
\hline $79^{\circ}$ & 0,13 & 1 & 0,19 & 1 \\
\hline $82^{\circ}$ & 0,44 & 1 & 0,04 & 1 \\
\hline $86^{\circ}$ & 0,36 & 2 & 0,06 & 1 \\
\hline $89^{\circ}$ & 0,55 & 4 & 0,1 & 2 \\
\hline $93^{\circ}$ & 0,22 & 3 & 0,1 & 2 \\
\hline $102^{\circ}$ & 0,06 & 2 & - & \\
\hline
\end{tabular}

Fonte: Autores (2021). 
Curiosamente, esse foi o período com maiores valores para densidade no sistema CAR (entre 1,16 a 1,92 indivíduos/gST), provavelmente, em virtude do alto teor de umidade (73,5 a 68,1\%) que favoreceu a incidência de espécies de dípteros, mas por outro lado, inibiu a ação de outros grupos, como os Mesostigmata, os quais são observados na faixa de 40 e $50 \%$ de umidade (Tabela 1). Esse resultado identifica que as espécies de mesoinvertebrados são influenciadas pela quantidade de água no sistema.

As observações in loco mostraram que o alto teor de umidade retardou o desenvolvimento da espécie Hermetia illucens no sistema CAR. Enquanto que o sistema CCR foram coletadas as larvas no estágio de segundo e terceiro instar, no sistema CAR só foi possível identificar as larvas de primeiro instar durante esse período de tempo.

Segundo Diener et al. (2011) durante a escassez de alimentos ou condições desfavoráveis, as larvas reduzem sua atividade metabólica ou deixam de se alimentarem. À medida que houve controle do teor de água, a espécie passou a se desenvolver normalmente.

No sistema CAR, observou-se que a partir do $30^{\circ}$ dia houve diminuição gradativa da densidade de mesoinvertebrados até chegar a valores poucos expressivos, devido à carência de alimento e ao baixo teor de umidade. Muitos mesoinvertebrados da ordem Díptera completaram o seu ciclo de vida e saíram do sistema de compostagem.

Considerando que os sistemas são desenvolvidos com materiais diferentes, pode-se entender que as composteiras exerceram influência sobre a dinâmica dos organismos. Essa situação também foi identificada na pesquisa de Delgado et al. (2015) ao avaliar o processo de compostagem de resíduos vegetais e estrume em dois sistemas de fardos e um sistema controle localizado a cerca de $100 \mathrm{~m}$ de distância. Segundo os autores, a quantidade e diversidade de artrópodes variaram entre os fardos e foram significativamente maiores que o controle. Ainda segundo os autores, o tipo de sistema de compostagem, a composição do substrato, as condições ambientais e a área em que é realizado o experimento influenciam diretamente nos organismos que participam da decomposição da matéria orgânica.

A integridade das comunidades de mesoinvertebrados encontrados no sistema de compostagem deve-se à análise do substrato de forma mais próxima da realidade gerada in loco. Assim, foi priorizado avaliar como as comunidades biológicas se comportam em um dado sistema na ausência de trituração do substrato, ausência de correção de pH, instalação de sistema em escala real e no uso da parcela orgânica conforme gerada pelos moradores.

Verificou-se que o cuidado de utilizar o substrato próximo ao gerado pelos moradores na área da pesquisa, foi favorável para análise concreta de dados referentes aos resíduos sólidos gerados na área de estudo e mostrou ser viável para ação metabólica dos mesoinvertebrados.

As alterações dos parâmetros físicos e químicos observadas nos sistemas estudados resultaram da ação dos organismos, das propriedades do substrato, do modelo das composteiras e das condições climáticas que indiretamente afetaram o seu funcionamento. As influencias diretas como temperatura ambiente e chuvas intensas foram amenizadas com a cobertura do sistema. Este resultado se mostrou fundamental à ação e ao desenvolvimento de várias espécies que apresentam limites de tolerância mais estreitos nessas fases.

Como o processo de compostagem é medido pelos organismos participantes do sistema, as alterações que ocorrem no meio físico afetam diretamente às populações e o seu desenvolvimento, sendo definidas por fatores bióticos e abióticos que atuam seletivamente nos organismos, como afirma Odum (2012).

A presença e o sucesso de um organismo ou de uma comunidade dependem de um conjunto de fatores limitantes, tais como, condições ambientais, o modelo de composteira, a composição da matéria orgânica, o tamanho das partículas e o teor de umidade, entre outros parâmetros, que podem alterar a comunidade de organismos no sistema, influenciando na qualidade final do composto (Silva, 2008; Silva et al., 2010; Delgado et al., 2015; Araújo et al., 2019; Freitas et al. 2020; Silva et al., 2020). 
Para Silva (2008), o controle dos parâmetros físico-químicos é essencial para ação sinérgica de um conjunto de organismos, fato comprovado durante a execução deste trabalho.

Compreende-se que a ação dos organismos aeróbios durante o tratamento dos resíduos sólidos orgânicos expressa as relações de um sistema ecológico, logo, ocorrem dentre outras, as cadeias tróficas, o ciclo da matéria, o fluxo de energia, sucessão ecológica e as atividades que permitem a homeostase do próprio sistema. Por conseguinte, favorece a continuidade das espécies, ao mesmo tempo em que soluciona um dos principais problemas que concorrem para o desequilíbrio ambiental.

Nessa perspectiva, identificar os níveis taxonômicos fundamentais para degradação da matéria orgânica, compreender a dinâmica entre os organismos e os níveis de tolerância é um meio de impulsionar o processo e melhorar a qualidade do produto final.

\subsection{Análise de correlação entre os mesoinvertebrados e os parâmetros monitorados nos sistemas CCR e CAR}

Os organismos que possuem larga faixa de tolerância para um parâmetro podem apresentar estreita faixa para outro fator ecológico. Outros, por sua vez, se distribuem ao longo de um amplo gradiente de tolerância, adaptando-se a diferentes condições físicas (Odum, 2012). Essa plasticidade ecológica permite que populações especialistas possam sobreviver em condições ambientais diversificadas.

Alguns princípios subsidiários aos fatores limitantes do meio necessitam ser investigados. Nessa ótica, a aplicação do Teste de Pearson mostrou-se uma alternativa adequada para avaliar a correlação dos mesoinvertebrados presentes nos sistemas estudados com os principais parâmetros monitorados (Tabela 2).

Tabela 2. Correlação entre os mesoinvertebrados e os parâmetros monitorados nos sistemas CCR e CAR. Campina Grande, estado da Paraíba, Brasil. Agosto e novembro de 2017.

\begin{tabular}{|c|c|c|c|c|}
\hline \multirow{3}{*}{ Parâmetros } & \multicolumn{4}{|c|}{ Correlação } \\
\hline & \multicolumn{2}{|c|}{ CCR } & \multicolumn{2}{|c|}{ CAR } \\
\hline & Valor de $\mathbf{r}$ & Classificação & Valor de $r$ & Classificação \\
\hline $\mathrm{pH}$ & 0,39 & Fraca & 0,35 & Fraca \\
\hline Umidade (\%) & 0,27 & Fraca & 0,42 & Moderada \\
\hline STV $(\%$ ST) & 0,26 & Fraca & 0,27 & Fraca \\
\hline $\mathrm{COT}(\% \mathrm{ST})$ & 0,26 & Fraca & 0,27 & Fraca \\
\hline Temperatura $\left({ }^{\circ} \mathrm{C}\right)$ & 0,22 & Fraca & 0,09 & Bem fraca \\
\hline
\end{tabular}

Fonte: Araújo (2018).

O teste revelou a existência de correlação positiva entre os mesoinvertebrados e as variáveis observadas ( $\mathrm{pH}$, teor de umidade, STV, COT e temperatura) durante a compostagem (Tabela 2), indicando a interdependência entre estes fatores e os mesoinvertebrados. Embora, tais relações oscilaram entre bem fraca e moderada, não obtendo correlações perfeitas.

A correlação positiva verificada para mesoinvertebrados e STV expressa à necessidade de observar a fração de matéria orgânica no decorrer do sistema e que os tratamentos seguem a tendência de decréscimo de STV à medida que a atividade metabólica se torna mais lenta, limitando a taxa cinética de degradação.

Os níveis de $\mathrm{pH}$ correlacionaram positivamente com mesoinvertebrados, mostrando que o ambiente é favorável para atuação destes. 
Em relação à temperatura em ambos os sistemas, notaram-se coeficiente de correlação classificada fraca em relação aos mesoinvertebrados, não representando interferência significativa para os sistemas estudados. Constata-se que nas condições estudadas, os níveis de temperatura não constituíram um fator determinante para a maioria desses organismos. Sabese, porém, que o aumento do número de organismos atuando na degradação da matéria orgânica, provoca a elevação dos níveis de temperatura no sistema, haja vista que prevalecem os organismos exotérmicos.

Os teores médios de umidade expressaram uma correlação positiva moderada para o sistema CAR, ratificando as observações realizadas in loco, principalmente nos dias em que as condições ambientais sofriam alterações significantes. O controle de umidade foi favorável à ação dos organismos no sistema, mas, à medida que ocorria diminuição ou aumento excessivo, a densidade de mesoinvertebrados era reduzida. Essa condição ficou bastante clara, especialmente para os grupos de ácaros observados no sistema.

Verificou-se que a correlação entre mesoinvertebrados e algumas das principais variáveis observadas na pesquisa se mostrou positiva, mas com classificações relativamente insignificantes à luz dos princípios estatísticos. Fato que pode ser atribuído à quantidade de organismos com faixas de tolerância diversificada. Enquanto uns se mostraram adaptados praticamente a todas as etapas do sistema, outros apareceram apenas em condições específicas. Sugere-se análise de cada grupo de organismo para identificação da correlação com as variáveis.

Os dados relativos à análise de correlação mostram que os sistemas constituem um processo biológico. Os mesoinvertebrados correlacionam positivamente com os parâmetros físico-químicos de forma que as interferências na cadeia alimentar geram a consequente procrastinação na degradação da matéria orgânica.

\section{Conclusão}

Nas condições estudadas, é possível inferir que os mesoinvertebrados são facilmente observados, a metodologia de investigação aplicada é economicamente acessível e o seu ciclo de vida parcialmente curto são características que permitem aos pesquisadores e às pesquisadoras o acompanhamento e a identificação desses organismos, favorecendo a tomada de decisão, no sentido de alcançar os objetivos pressupostos ao tratamento dos resíduos sólidos orgânicos, sanitização e higienização.

A densidade e a diversidade de mesoinvertebrados revelam o seu papel biológico e nicho ecológico em relação à ciclagem da matéria orgânica e à transformação eficiente de energia ao longo de seu fluxo.

Ao passo que degradam a matéria orgânica, os mesoinvertebrados exercem importante função ao triturar a matéria orgânica e convertendo-a em biomassa, beneficiando a ação de microrganismos; são indicadores das condições físicas e químicas do sistema; promovem o controle de vetores e de microrganismos com interesse médico e contribuem para obtenção de composto orgânico com características sanitárias e agronômicas viáveis a diferentes culturas.

Considera-se inviável direcionar apenas aos microrganismos a função de degradação da matéria orgânica em sistemas de compostagem, porque ao longo desse processo há participação efetiva de uma diversidade de mesoinvertebrados que desempenha diferentes nichos ecológicos ao longo de todas as etapas da compostagem.

Ratifica-se que a presença e o sucesso de um organismo ou de uma comunidade dependem de um conjunto de fatores limitantes, tais como, condições ambientais, o modelo de composteira, a composição da matéria orgânica, o tamanho das partículas e o teor de umidade, entre outros parâmetros, que podem alterar a comunidade de organismos no sistema, influenciando na qualidade final do composto. 
Garantir condições favoráveis aos diferentes organismos autotócnes para o desempenho do seu nicho ecológico é essencial ao alcance dos objetivos da compostagem: estabilização e sanitização dos resíduos sólidos orgânicos. À medida que são dadas condições a estes organismos, são propiciadas condições inóspitas aos patogênicos.

Recomenda-se a ampliação deste tipo de estudo para outros grupos de organismos, tais como bactérias e fungos, visando favorecer o tratamento de resíduos sólidos orgânicos domiciliares e reduzir ou evitar os diferentes impactos negativos provocados por estes resíduos quando são destinados e dispostos de forma errada. Pode constituir uma opção para transformar problema em solução, considerando-se a sua composição em termos de nutrientes necessários aos sistemas edáficos. Constitui ainda uma ação que pode contribuir de forma efetiva para o alcance dos objetivos previstos para gestão integrada de resíduos sólidos. Ressalta-se, no entanto, que a gestão de resíduos sólidos requer o processo de educação ambienal conforme prevê a legislação ambiental brasileira.

\section{Referências}

Araújo, E. C. S. (2018). Organismos que participam das diferentes fases do tratamento aeróbio de resíduos sólidos orgânicos domiciliares. Dissertação (Mestrado em Ciência e Tecnologia Ambiental). Campina Grande-PB: Universidade Estadual da Paraíba, 175p.

Araújo, E. C. S., Silva, M. M. P., Silva, A. V., Santos Sobrinho, J. B. \& Leite, V. D. (2019). Tratamento aeróbio de resíduos sólidos orgânicos domiciliares em sistemas descentralizados móveis. Anais. $30^{\circ}$ Congresso Brasileiro de Engenharia Sanitária e Ambiental. ABES: Natal-RN.

Araújo, E. C. S., Silva, M. M. P., Silva, A. V., Leite, V. D. \& Barros, A. T. (2019). Diversidade e funções de mesoinvertebrados no tratamento aeróbio de resíduos sólidos orgânicos domiciliares em sistemas descentralizados. Anais. $30^{\circ}$ Congresso Brasileiro de Engenharia Sanitária e Ambiental. ABES: Natal-RN.

Brasil. Resolução Conama $n^{o} 481$, de 03 de outubro de 2017. (2017). Estabelece critérios e procedimentos para garantir o controle e a qualidade ambiental do processo de compostagem de resíduos orgânicos, e dá outras providências. Brasília-DF: Ministério do Meio Ambiente.

Borror, D. J \& Delong, D. M. (1992). An Introduction to the study of Insects. Sixth edition, Harcourt Brace Janovich College Publishers, 876 p.

Delgado, L. J. A., Córdola, J. C., Pérez, G.S. \& Arango, Y. L. (2015). Decomposición de resíduos orgánicos en pacas: aspectos fisicoquímicos, biológicos, ambientales y sanitários. Revista producción + limpia, v. 10 (2), 38-52.

Diener, S., Zurbrügg, C., Gutiérrez, F. R., Koottatep, D. H. N., Morel, A. \& Tockner, K. (2011). Black soldier fly larvae for organic waste treatment prospects and constraints. Proceedings of the Waste Safe 2011: Khulna, Bangladesh.

Freitas, A. F., Leite, V. D., Silva, M. M. P., Gomes, R. B., Silva, M. J., Farias, F. L. B. \& Barros, A.T. (2020). Tratamento aeróbio de resíduos sólidos orgânicos gerados em condomínio vertical como alternativa sustentável. Research, Society and Development, 9(10), 1-27.

Gilbert, J., Ricci-Jürgensen, M. \& Ramola, A. (2020). Benefits of compost and anaerobic digestate; when applied to soil. https://www.iswa.org/uploads/media/Report_2_Benefits_of_Compost_and_Anaerobic_Digestate_01.pdf.

Jürgensen, M. R., Gilbert, J. \& Ramola, A. (2020). Global assessment of municipal organics waste production and recycling. Recuperado de https://www.iswa.org/uploads/media/Report_1_Global_Assessment_of_Municipal_Organic_Waste_Compressed_v2.pdf.

Kaza, S., Yao, L., Bhada-Tata, P. \& Woerden, F. V. (2018). What Waste 2.0: A global snapshot of solid waste management to 2050. World bank group.

Madigan, M. T., Martinko, J. M., Bender, K. S., Buckley, D. H. \& Stahl, D. A. (2016). Microbiologia de Brock. Porto Alegre’'RS: ArtMed. 1032p.

Mcalpine, J. F., Peterson, B. V., Shewell, G. E., Teskey, H. J., Vockeroth, J. R. \& Wood, D. M. (1987). Manual of Nearctic Diptera. Research Branch Agriculture. Canada, 2 (28), 684.

Morales, G. E. \& Wolff, M. (2010). Insects associated with the composting processo of solid urban waste separated at the source. Revista Brasileira de Entomologia, 54 (4), 645-653.

Mira, G. M. \& Jaramillo, C. A. P. (2010). Evaluación cinética de los dípteros como indicadores de la evolución del proceso de compostaje. Revista ingenieria universidad de Medellin, 9 (17), 13-28.

Nascimento, C. R. (2015). Sistema de tratamento descentralizado de resíduos sólidos orgânicos no bairro Malvinas, Campina Grande-PB. Dissertação (Mestrado em Ciência e Tecnologia Ambiental). Campina Grande-PB: Universidade Estadual da Paraíba. 110p.

Nascimento, C. R., Silva, M. M. P., Araújo, E. C. S., Costa, M. P. \& Silva, A. V. (2017). Avaliação de sistema de tratamento aeróbio descentralizado móvel de resíduos sólidos orgânicos domiciliares no bairro Malvinas, Campina Grande-PB. Anais. $29^{\circ}$ Congresso Brasileiro de Engenharia Sanitária e Ambiental. ABES: São Paulo-SP.

Odum, E. P. (2012). Ecologia. Rio de Janeiro: Guanabara. 434p.

Polprasert, C. \& Koottatep, T. (2017). Organic Waste Recycling: Technology, Management and Sustainability. London: IWA Publishing. 
Research, Society and Development, v. 10, n. 1, e59710112173, 2021

(CC BY 4.0) | ISSN 2525-3409 | DOI: http://dx.doi.org/10.33448/rsd-v10i1.12173

Shimakura, S. E. (2006). Correlação; interpretação do coeficiente de correlação. http://leg.ufpr.br/ silvia/CE003/node74.html.

Silva, M. M. P. (2008). Tratamento de lodos de tanques sépticos por co-compostagem para municípios do semi-árido paraibano: alternativa para mitigação de impactos ambientais. Tese de Doutorado, Universidade Federal de Campina Grande, Campina Grande, Paraíba, Brasil. 291 p.

Silva, M. M. P., Sousa, J. T., Ceballos, B. S. O., Feitosa, W. B. S. \& Leite, V. D. (2010). Avaliação sanitária de resíduos sólidos orgânicos domiciliares em municípios do semiárido paraibano. Revista Caatinga, Mossoró-RN, 23(2), 87-92.

Silva, M. M. P., Gomes, R. B., Araújo, E. C. S., Gomes, I., Freitas, A. F., Silva, A. V., Farias, F. L. B. \& Leite, V. D. (2020). Prevalência de helmintos em resíduos sólidos orgânicos domiciliares; um risco à saúde ambiental e humana. Brazilian Journal of Development, 6 (5), 28689 -28702.

Silva, M. M. P., Lima, R. A., Gomes, R. B., Mendes, R. A., Santos Sobrinho, J. B., Araújo, E. C. S. \& Lima, V. G. S. (2020a). Educação Ambiental: ferramenta indispensável à gestão municipal de resíduos sólidos. Brazilian Journal of Development, 6 (5), $28743-28757$.

Stehr, F. W. (1991). Immature insects. Dubuque, Kendall/Hunt.

Steel, H. \& Bert, W. (2011). Biodiversity of compost mesofauna and its potential as an indicator of the composting process status. Dynamic soil, dynamic plant, v.5 (2), 45-50.

Tingle, F. C., Mitchell, E. R., \& Copeland, W. W. (1975). The soldier fly, Hermetia illucens, in poultry houses in north central Florida. Journal of the Georgia Entomological Society, 10, 179-183. 